\title{
Des doutes insuffisants pour changer nos pratiques
}

Ursula Winterfelda, David Baud ${ }^{b}$, Alice Panchauda, Laura Rothuizen ${ }^{a}$, Françoise Livioa, Rudolf Stollerc, Thierry Buclin $^{a}$

a Swiss Teratogen Information Service, Division de Pharmacologie clinique, CHUV, Lausanne; ${ }^{b}$ Unité de recherche en Médecine materno-foetale, Département de Gynécologie-Obstétrique, Maternité, CHUV, Lausanne; ${ }^{\mathrm{c}}$ Swissmedic, Schweizerisches Heilmittelinstitut, Bern

En 2014, les médias ont diffusé l'information d'un lien suggéré entre la prise de paracétamol par la mère pendant la grossesse et la survenue ultérieure de troubles du développement psychomoteur et de déficit de l'attention avec ou sans hyperactivité (TDAH) chez l'enfant. Or les résultats des études publiées se sont révélés insuffisants pour confirmer ce risque. Ainsi, ces données ne justifient pas une remise en question de la place thérapeutique du paracétamol chez la femme enceinte.

Plusieurs études récentes ont rapporté une association entre l'exposition in utero au paracétamol et un risque accru de troubles du développement psychomoteur et de TDAH. Les résultats de ces études ont été largement repris et interprétés par les médias dans des communiqués souvent alarmistes, avec pour conséquence probable la diffusion d'une perception exagérée du risque chez les professionnels de santé et les patientes.

\section{Un signal de tératovigilance intéressant - sans lien de cause à effet confirmé}

Une première étude de cohorte prospective publiée en 1987 n'a pas rapporté d'impact d'un traitement de paracétamol pendant la grossesse sur le QI ou sur l'attention des enfants suivis jusqu'à l'âge de quatre ans [1]. Il s'agissait d'une cohorte de 1529 femmes enceintes dont $41 \%$ avaient pris du paracétamol pendant la première moitié de la grossesse. Trois études publiées entre 2013 et 2014 et détaillées ci-dessous ont en revanche décrit une association entre l'exposition in utero au paracétamol et des troubles du développement psychomoteur ou des TDAH [2-4].

Une étude de cohorte norvégienne portant sur 48631 grossesses a analysé des différences de développement psychomoteur chez 2919 frères ou sœurs dont un seul

Centre d'information dédié aux professionnels de santé en Suisse

Une observation d'exposition médicamenteuse problématique pendant la grossesse ou l'allaitement? Un doute sur l'attitude à suivre? Contactez le centre d'information "médicament grossesse et allaitement»: Swiss Teratogen Information Service - STIS, 1011 Lausanne-CHUV, Tél. 02131442 67, www.swisstis.ch avait été exposé au paracétamol pendant la grossesse [2]. Les mères ont évalué le développement psychomoteur de leurs enfants à l'âge de trois ans en utilisant différents questionnaires et échelles validés. L'utilisation de paracétamol pendant 28 jours ou plus au cours de la grossesse a été associée à une augmentation de risque d'altération du développement psychomoteur chez les enfants exposés, notamment de la motricité, de la communication, du comportement et de l'activité. En retenant une prévalence de 6\% du TDAH et de troubles psychomoteurs dans la population pédiatrique générale, les résultats de cette étude correspondraient à un risque relatif de 1,7 soit grossièrement à un risque absolu de $10 \%$ en cas d'exposition. Une exposition au paracétamol de moins de 28 jours avait également un impact négatif sur la motricité, mais aucun effet sur toutes les autres échelles mesurées.

Une étude de cohorte danoise portant sur 64322 enfants a analysé l'association entre l'exposition in utero au paracétamol et le risque de TDAH. Plus de la moitié des mères ont déclaré avoir pris du paracétamol pendant la grossesse. Les cas de TDAH et autres troubles apparentés ont été répertoriés sur les bases suivantes: évaluation parentale du comportement de l'enfant à l'âge de sept ans (questionnaire validé); diagnostic de TDAH identifié dans l'un ou l'autre des deux registres de santé consultés; traitement médicamenteux du TDAH identifié dans un registre de prescription. Plusieurs facteurs confondants ont été pris en considération (infection ou pathologie inflammatoire pendant la grossesse, santé mentale maternelle). L'utilisation de paracétamol a été associée à une augmentation du risque 
de comportement hyperactif à l'âge de sept ans (risque relatif ou risk ratio 1,13, IC 95\% 1,01-1,27), de diagnostic de TDAH (rapport de risque ou hazard ratio [HR] 1,37, IC 95\% 1,19-1.59) et de prescription d'un traitement médicamenteux du TDAH (HR 1,29, IC 95\% 1,15-1,44) [3]. L'association tendait à être plus marquée lors d'exposition prolongée.

\section{Le Paracétamol reste l'antalgique de première intention pendant la grossesse.}

Enfin, une étude de cohorte néo-zélandaise portant sur 871 enfants a analysé l'association entre l'utilisation de différents médicaments pendant la grossesse (paracétamol, aspirine, antiacides, antibiotiques) et la survenue de troubles du comportement ou de symptômes de TDAH à l'âge de sept et onze ans [4]. Les auteurs ont tenu compte d'un certain nombre de facteurs confondants (poids de naissance, statut socio-économique, stress maternel anténatal). L'évaluation de la santé mentale des enfants a été réalisée par les parents et les enfants eux-mêmes, à l'aide de questionnaires et d'échelles validées. Près de la moitié $(49,8 \%)$ des mères avaient rapporté une utilisation de paracétamol pendant la grossesse. Les scores se sont révélés légèrement moins bons chez les enfants exposés, avec une différence de l'ordre de 1 point sur une échelle de 40 points.

\section{Prudence dans l'interprétation des résultats}

Plusieurs éléments sont sujets à caution dans l'interprétation de ces études. Les associations observées dans les deux études scandinaves sont à la limite de la signification statistique alors que des milliers de patients ont été inclus, et la différence de score était de très faible ampleur, cliniquement peu relevant, dans la $3^{\mathrm{e}}$ étude. La plupart des résultats se basent sur un dépistage parental de modification du comportement des enfants à l'aide de différents questionnaires et échelles, sans validation des diagnostics par des professionnels de santé. Dans les deux études scandinaves, les associations tendaient à être plus marquées lors d'exposition prolongée au paracétamol (>28 jours; plus d'un trimestre). Une telle utilisation du paracétamol pendant la grossesse étant inhabituelle, il ne peut être exclu qu'une pathologie maternelle ait joué un rôle dans les résultats observés. Les troubles du développement psychomoteur et le TDAH sont en effet d'origine multifactorielle. Des facteurs confondants potentiels (troubles somatiques ou psychiques maternels, exposition in utero à de l'alcool ou à des substances illicites, exposition des enfants à divers facteurs environnementaux pendant les premières années de vie, etc.) pourraient donc être associés à la fois à la prise de paracétamol par la mère et à la survenue de TDAH chez l'enfant, même si les études ont appliqué des ajustements pour certaines variables. L'exposition en termes de doses de paracétamol étant inconnue dans ces études, il existe un doute sur l'ampleur réelle de l'exposition maternelle et par conséquent sur l'interprétation d'un effet dose-réponse. En résumé, ces études rapportent une faible association entre l'exposition in utero au paracétamol et le risque de troubles du développement psychomoteur et de TDAH, sans pour autant démontrer un lien de causalité.

\section{Ces nouvelles données ne remettent pas en cause la place thérapeutique du paracétamol chez la femme enceinte}

Les observations récemment rapportées étant insuffisamment étayées pour remettre fondamentalement en cause son utilisation telle que pratiquée jusqu'ici, le paracétamol reste l'antalgique de première intention pendant la grossesse. Le profil de sécurité d'alternatives thérapeutiques telles que les AINS reste moins favorable que celui du paracétamol. De même, ces données ne justifient pas le remplacement du paracétamol par des opioïdes pour la prise en charge de douleurs faibles à modérées pendant la grossesse. Toutefois, il n'est presque jamais possible d'affirmer formellement qu'il n'existe aucun potentiel délétère pour l'enfant d'une exposition in utero à un médicament. Ceci est particulièrement vrai dans des situations où la prise médicamenteuse sort du contexte dans lequel la majorité des données de sécurité chez la femme enceinte ont été récoltées, tel que lors de dépassement de doses ou de durées usuelles de traitement. Un rappel de principe d'une utilisation parcimonieuse de tout médicament chez la femme enceinte reste ainsi à préconiser.

Nous rappelons qu'il faut annoncer les effets indésirables suspectés des médicaments (EI), de préférence en utilisant le système électronique ElViS ou le formulaire papier, cf. www.swissmedic.ch

\section{Références}

1 Streissguth AP, Treder RP, Barr HM, Shepard TH,Bleyer WA, Sampson PD, Martin DC. Aspirin and acetaminophen use by pregnant women and subsequent child IQ and attention decrements. Teratology. 1987;35:211-9.

2 Brandlistuen RE, Ystrom E, Nulman I,Koren G, Nordeng H. Prenatal paracetamol exposure and child neurodevelopment: a sibling controlled cohort study. Int J Epidemiol. 2013;42:1702-13.

3 Liew Z, Ritz B, Rebordosa C, Lee PC, Olsen J. Acetaminophen use during pregnancy, behavioral problems, and hyperkinetic disorders. JAMA Pediatr. 2014;168:313-20.

4 Thompson JM, Waldie KE, Wall CR, Murphy R, Mitchell EA; ABC study group. Associations between acetaminophen use during pregnancy and ADHD symptoms measured at ages 7 and 11 years. PLoS One. 2014;9:e108210. 